\title{
Caracterización poscosecha del fruto de durazno [Prunus persica (L.) Batsch] cv. Dorado producido bajo condiciones de trópico alto
}

\section{Postharvest characterization of peach fruit [Prunus persica (L.) Batsch] cv. Dorado produced under high-altitude tropical conditions}

KAREN LISSETH AFRICANO'

PEDRO JOSÉ ALMANZA-MERCHÁN'

HERNANDO CRIOLLO E. ${ }^{2}$

ANÍBAL HERRERA ${ }^{3}$

HELBER ENRIOUE BALAGUERA-LÓPEZ ${ }^{1,4,5}$

Fruto de durazno cv. Dorado en madurez de consumo.

Foto: E.H. Pinzón S.

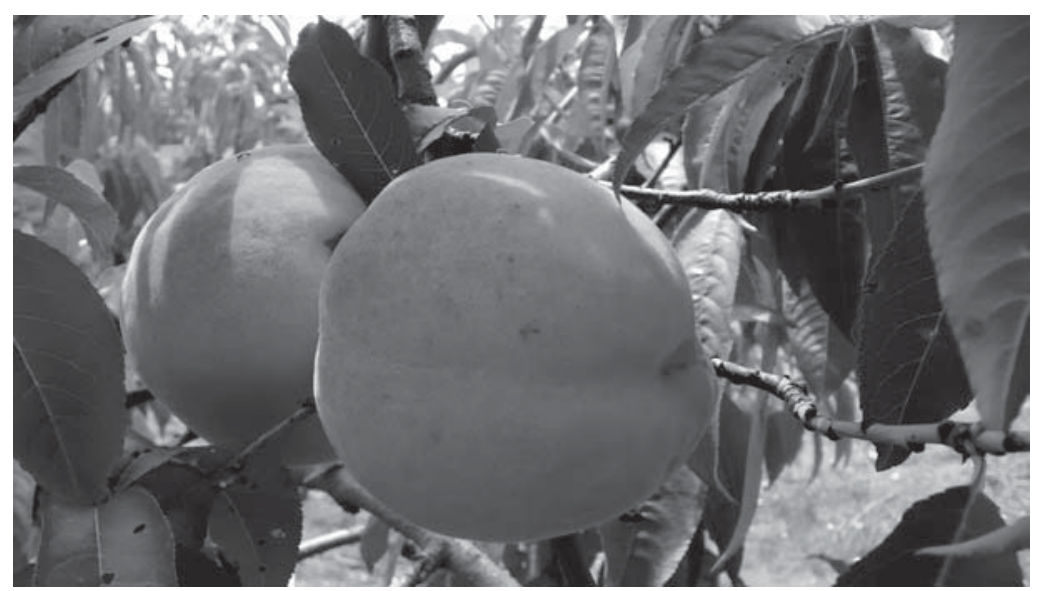

\section{RESUMEN}

El durazno pertenece a la familia Rosaceae; su fruto es una drupa muy apetecida por su agradable sabor, aroma y características nutricionales. Es un fruto climatérico, altamente perecedero con elevada intensidad respiratoria y producción de etileno. Con el fin de realizar la caracterización poscosecha del fruto de durazno cv. Dorado bajo condiciones del trópico alto, se cosecharon frutos en el municipio de Tuta (Boyacá, Colombia) en madurez fisiológica. Se realizaron seis mediciones cada tercer día de parámetros fisicoquímicos como: pérdida de peso, color de epidermis y pulpa, tasa respiratoria, firmeza, extensibilidad, sólidos solubles totales (SST) y acidez total titulable (ATT). El fruto de durazno presentó un comportamiento climatérico de acuerdo con su tasa respiratoria; alcanzando el pico climatérico a los 9 días de almacenamiento, la pérdida de peso aumentó linealmente, con un valor de 17,18\% en el día 11 de almacenamiento (temperatura de $18 \pm 2^{\circ} \mathrm{C}$ y humedad relativa de $75 \pm 5 \%$ ), la firmeza disminuyó durante el almacenamiento alcanzando valores de 6,5 N, de igual manera la ATT disminuyó a medida que avanzó la maduración del fruto; pasando de 0,95\% en el día 1 a 0,76\% en el día 11 de almacenamiento. Por su parte, el índice de color de la epidermis y de la pulpa, los SST, la relación de madurez (RM) y la extensibilidad aumentaron durante la poscosecha.

\footnotetext{
Facultad de Ciencias Agropecuarias, Programa de Ingeniería Agronómica, Grupo de Investigaciones Agrícolas (GIA), Universidad Pedagógica y Tecnológica de Colombia (UPTC), Tunja (Colombia).

2 Facultad de Ciencias Agrarias, Grupo de Investigación Producción de Frutales Andinos, Universidad de Nariño, Pasto (Colombia).

3 Facultad de Ciencias Agrarias, Departamento de Agronomía, Universidad Nacional de Colombia, Bogotá (Colombia).

4 Facultad de Ciencias, Programa de Biología, Universidad El Bosque, Bogotá (Colombia).

5 Autor para correspondencia. helber.balaguera@uptc.edu.co
} 
Palabras clave adicionales: almacenamiento, climaterio, maduración.

\section{ABSTRACT}

Peach belongs to the Rosaceae family; its fruit is a drupe highly appreciated for its pleasant flavour, aroma, and nutritional characteristics. It is also a highly perishable climacteric fruit with elevated respiratory rates and ethylene production. In order to characterize postharvest behavior of peach cv. Dorado fruits under high tropic conditions, fruits at physiological maturity were harvested in the municipality of Tuta (Boyacá, Colombia). The following physicochemical parameters were measured six times, every third day: weight loss, skin and pulp color, respiration rate, firmness, extensibility, total soluble solids (TSS), and total titratable acidity (TTA). In accordance with the respiration rate, peach fruits showed a climacteric behavior; reaching a climacteric peak at the ninth day of storage, weight loss increased linear with the time, reaching $17.18 \%$ on day 11 of storage (temperature: $18 \pm 2^{\circ} \mathrm{C}$, relative humidity: $75 \pm 5 \%$ ). Firmness decreased along the storage period achieving values of $6.5 \mathrm{~N}$, in a similar way, TTA declined with the advance of the fruit ripeness stage; falling from $0.95 \%$ on day 1 to $0.76 \%$ on day 11 of storage. Moreover, skin and pulp color indexes, TSS, maturity ratio and extensibility increased during postharvest.

Additional key words: storage, climacteric, ripening.

Fecha de recepción: 10-07-2016 Aprobado para publicación: 24-11-2016

INTRODUCCIÓN

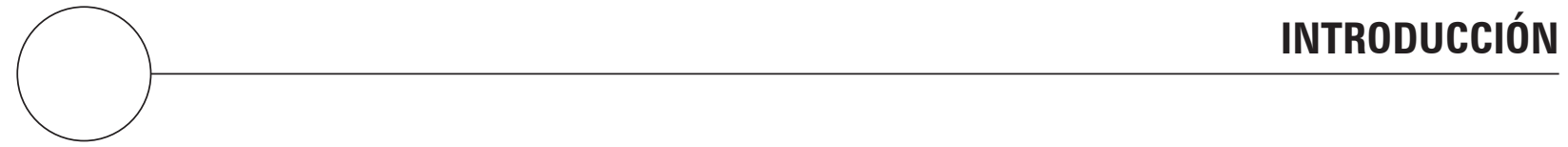

El durazno [Prunus persica (L.) Batsch] es un frutal caducifolio, originario de China y perteneciente al género Prunus de la familia Rosaceae (Castro y Puentes, 2012). En Colombia, es considerado uno de los frutales de mayor importancia en las zonas de trópico alto, ya que posee ventajas comparativas con los que se siembran en las zonas templadas (Pinzón et al., 2014). Estas ventajas están relacionadas con la ubicación geográfica de Colombia en el trópico y la diversidad de microclimas que existen, que junto con un buen manejo agronómico, oferta agroecológica y el uso de variedades tempranas, tardías e intermedias se pueden producir para el consumo en fresco y la agroindustria todo el año (Castro y Puentes, 2012; Fischer et al., 2010). Para el año 2013 el área cosechada en durazno en el país fue de 1.804 ha, con una producción de 25.584 t y un rendimiento de 14,2 t hasiendo Boyacá el departamento con mayor participación en la producción nacional $(52,27 \%)$ con un área cosechada de 828,20 ha y 12.952 t (Agronet, 2016). El sistema de producción de frutales caducifolios en Boyacá se enmarca básicamente en el subsector de la economía campesina, con predominio de pequeñas agroempresas con extensiones alrededor de 2,8 ha (Puentes et al., 2008).
En Boyacá se encuentran las siguientes variedades de durazno: Rey negro, Rubidoux, Dorado, Diamante, Jarillo y Granjarillo (Castro y Puentes, 2012). Dentro de estas, el cv. Dorado se caracteriza porque se adapta entre los 2.200 a $2.700 \mathrm{msnm}$, presenta piel amarilla con ligera pigmentación roja, pulpa amarilla oro con coloración rojiza al rededor del hueso, presenta forma redondeada, con sutura levemente desarrollada, con un peso promedio de $150 \mathrm{~g}$ y posee buenas características para la industrialización y para el consumo en fresco (Campos, 2013). Estas características, sumadas a su bajo requerimiento de horas frío y al rápido desarrollo del fruto la convierten junto con la variedad Diamante en las predominantes en los departamentos de Boyacá, Cundinamarca y parte del Huila (Miranda y Carranza, 2013).

En general, el fruto de durazno, por su agradable sabor y propiedades nutricionales, tiene gran aceptación por parte de los consumidores (Lambaré y Pochettino, 2012). El consumo de este fruto aporta vitamina $A$, B1, B2, C, fósforo, calcio entre otros minerales y vitaminas esenciales (Herrera et al., 2006). El durazno presenta un comportamiento climatérico (Africano et al., 2015), siendo el etileno el responsable de regular 
los principales procesos moleculares, bioquímicos y fisiológicos durante la maduración en este fruto, incluyendo el incremento en la intensidad respiratoria y en los sólidos solubles totales (Akbudak y Eris, 2004), cambios de color a nivel de epidermis y pulpa, disminución de la acidez total titulable y de la firmeza de la pulpa (Altube et al., 2001). Sin embargo, es importante mencionar que variables como la firmeza son dependientes de la variedad, al igual que el contenido de sólidos solubles, que además se ve influenciado por la temporada, la ubicación y la fecha de recolección (Ruiz-Altisent et al., 2006).

A pesar de que se desconoce el comportamiento fisicoquímico durante el periodo de poscosecha del cv. Dorado en las zonas productoras de trópico alto colombiano, existen estudios sobre la calidad poscosecha de diferentes variedades de durazno en otras zonas. Al respecto, Weber et al. (2003) y García (2006) realizaron la caracterización fisicoquímica durante la maduración de frutos de durazno 'Flordaking' y de tipo amarillo, respectivamente; Toncovich et al. (2008) estudiaron la calidad poscosecha de las variedades Start Lite, Alexandra, Aniversario, Caldessi 2000, María Bianca, Santa Lucía, Forastero y June Gold. Así mismo, Liu et al. (2015) evaluaron el comportamiento poscosecha del fruto de durazno 'Yuhualu' por medio de la determinación de la firmeza y cuantificación de la tasa respiratoria, producción de etileno, sólidos solubles totales y acidez total titulable, mientras que Infante et al. (2011) realizaron una caracterización físicoquímica de las variedades Elegant Lady y Carson.

La alta perecebilidad del fruto de durazno generada por la alta actividad metabólica del fruto y el alto porcentaje de agua (Herrera et al., 2006; Kays y Paull, 2004), junto con el desconocimiento del comportamiento fisicoquímico durante el periodo de poscosecha y el almacenamiento inadecuado, se pueden considerar como las principales causas de las pérdidas poscosecha en esta especie, al respecto, Castro y Puentes (2012) mencionan que existe un gran vacío en el manejo poscosecha, aspecto que le resta competitividad a este cultivo. Por tanto, el objetivo de esta investigación fue realizar la caracterización poscosecha del fruto de durazno cv. Dorado, producido bajo condiciones del trópico alto, con el fin de obtener más información para mejorar el almacenamiento de esta especie en Colombia.

\section{MATERIALES Y MÉTODOS}

El estudio se realizó en el laboratorio de Poscosecha de la Facultad de Ciencias Agrarias de la Universidad Nacional de Colombia, Bogotá. Como material vegetal se utilizaron frutos de durazno (Prunus persica (L.) Batsch) cv. Dorado, recolectados de un cultivo comercial ubicado en el municipio de Tuta (Boyacá, Colombia) en estado de madurez fisiológica. Se seleccionaron frutos en condiciones fitosanitarias óptimas, con tamaño y color homogéneos y sin evidencia de daños mecánicos. Posteriormente, se seleccionaron 40 frutos al azar y se dividieron aleatoriamente en 4 grupos para la caracterización durante la poscosecha. Finalmente, los frutos se mantuvieron en bandejas plásticas a temperatura ambiente (condiciones de laboratorio; temperatura de $18 \pm 2^{\circ} \mathrm{C}$ y humedad relativa de $75 \pm 5 \%$ ). Las características fisicoquímicas de los frutos al inicio del experimento se presentan en la tabla 1.

\begin{tabular}{|c|c|c|}
\hline bla 1. & $\begin{array}{l}\text { Parámetros fisicoq } \\
\text { no cv. Dorado al in } \\
\text { desviación estánda }\end{array}$ & $\begin{array}{l}\text { los frutos de duraz- } \\
\text { erimento }(n=10, \pm\end{array}$ \\
\hline & Parámetro & Valor \\
\hline Peso (g) & & $181,07 \pm 6,6$ \\
\hline Índice de & color & $-0,49 \pm 0,21$ \\
\hline Firmeza & & $12,81 \pm 1,2$ \\
\hline Relación & le madurez (SST/ATT) & $12,41 \pm 0,67$ \\
\hline
\end{tabular}

A los frutos cosechados se les realizaron mediciones cada tercer día y durante once días de almacenamiento, momento en el que los frutos perdieron la calidad comercial. Los parámetros fisicoquímicos medidos fueron los siguientes: tasa respiratoria, de acuerdo con la metodología utilizada por Balaguera-López et al. (2016), para lo cual, los frutos fueron ubicados en cámaras herméticas de $2 \mathrm{~L}$, conectadas a un sensor infrarrojo de $\mathrm{CO}_{2}$ y a un sistema de interfase y recolección de datos (Vernier, Beaverton, OR); cada 4 s y durante 5 min se registraron los valores de $\mathrm{CO}_{2}$, con estos valores se calculó la pendiente, que correspondió a la tasa respiratoria, se tuvo en cuenta el peso de los frutos y el volumen de la cámara para convertir los datos a mg de $\mathrm{CO}_{2} \mathrm{~kg}^{-1} \mathrm{~h}^{-1}$; pérdida de peso (\%) con la medición de peso fresco en balanza (Ohaus, Ohio, $\mathrm{OH}$ ) de precisión con aproximación de $0,01 \mathrm{~g}$ y por medio de la ecuación 1. El índice de color (IC, ecuación 2) se calculó a partir de parámetros del 
sistema CIELab L*, a* y b*, para lo cual se realizaron tres lecturas en el diámetro ecuatorial de cada fruto (Balaguera-López et al., 2014) con colorímetro digital CR-410 (Konica Minolta, Tokyo).

$$
\text { Pérdida de peso }(\%)=((\mathrm{P} 1-\mathrm{P} 2) / \mathrm{P} 1) \times 100
$$

Donde, P1: peso de frutos a tiempo inicial y P2: peso de frutos a tiempo final.

$$
\mathrm{IC}=\left(1000 \times \mathrm{a}^{*}\right) /\left(\mathrm{L}^{*} \times \mathrm{b}^{*}\right)
$$

La firmeza del fruto $(\mathrm{N})$ y extensibilidad de la epidermis $(\mathrm{mm})$ fueron determinadas mediante un texturómetro LS1 (marca Lloyd LS1, Bognor Regis, UK), acoplado al Software NexyGen PlusTM. El diámetro de la punta utilizada fue de $3 \mathrm{~mm}$. La firmeza correspondió a la máxima fuerza registrada por el equipo, mientras que la extensibilidad fue la distancia de deformación de la epidermis desde el momento en que la punta toca el fruto, hasta que se alcanza la máxima fuerza. Sólidos solubles totales (SST) se cuantificaron a través de la medición de ${ }^{\circ}$ Brix, con un refractómetro digital (marca Hanna, Woonsocket, RI) de rango 0 a $85 \%$ con precisión $0,1^{\circ} \mathrm{Brix}$. La acidez total titulable (ATT;\% de ácido málico) se calculó mediante titulación ácido base con $\mathrm{NaOH} 0,1 \mathrm{~N}$, utilizando la fórmula reportada por Rozo-Romero et al. (2015), y la relación de madurez (RM) por medio de la relación SST/ATT.

Con los datos obtenidos se realizó un análisis descriptivo, en el cual se calculó el promedio y el error estándar, también se ajustaron regresiones al comportamiento de los parámetros medidos. Se utilizó el software estadístico SAS v. 9.2.

\section{RESULTADOS Y DISCUSIÓN}

La tasa respiratoria de los frutos de durazno 'Dorado' mostró un comportamiento típico de un fruto climatérico, con intensidad respiratoria baja en los primeros días de almacenamiento, posteriormente se observó un incremento considerable alcanzando el pico climatérico en el día 9 de almacenamiento, seguido de un descenso que corresponde con el inicio de la fase de senescencia (Fig. 1A). Los resultados se ajustan con lo reportado por varios autores, quienes indican que el fruto de durazno es catalogado como un fruto climatérico, debido a que presenta un incremento en su tasa respiratoria después de que alcanza la madurez fisiológica y se prolonga hasta la senescencia (Kader, 2002; Tonetto de Freitas et al., 2007). De acuerdo con Azcón-Bieto y Talón (2013) los frutos climatéricos aumentan la tasa respiratoria durante la maduración para producir la energía que se requiere en el proceso de hidrólisis del almidón que conlleva a la síntesis de monosacáridos principalmente, como glucosa y fructosa.

El pico climatérico $\left(73,3 \pm 7,07 \mathrm{mg} \mathrm{CO}_{2} \mathrm{~kg}^{-1} \mathrm{~h}^{-1}\right)$ se observó en el día 9 de almacenamiento, mostrando un comportamiento similar al reportado para el cv. Yuhualu que alcanzó el climaterio en el día 10 de almacenamiento (Liu et al., 2015), estos resultados son un indicativo de la corta vida poscosecha del fruto de algunos cultivares. De acuerdo con Brovelli et al. (1999),
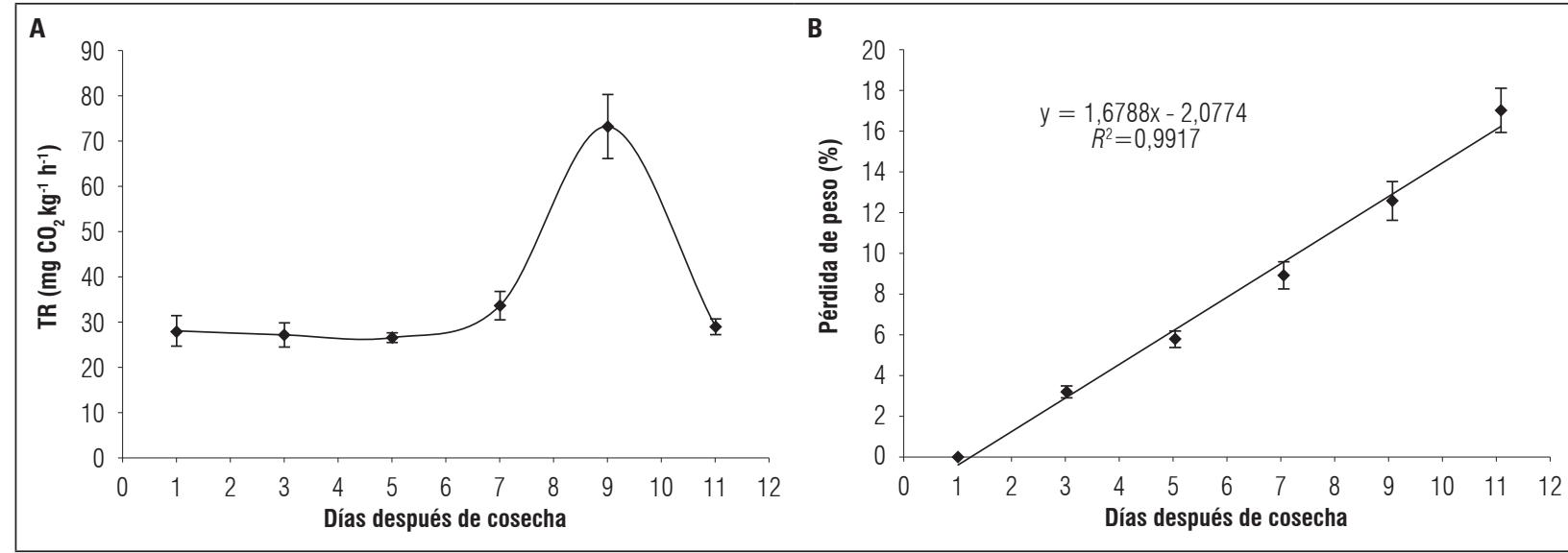

Figura 1. Comportamiento de la tasa respiratoria (A) y de la pérdida de peso (B) durante la poscosecha del fruto de durazno [Prunus persica (L.) Batsch] cv. Dorado. Los valores corresponden al promedio $(n=4)$. Las barras verticales corresponden al error estándar. 
durante la maduración el durazno presenta una tasa respiratoria moderada con valores entre 59 a $102 \mathrm{mg}$ $\mathrm{CO}_{2} \mathrm{~kg}^{-1} \mathrm{~h}^{-1}$, lo que coincide con lo encontrado en el cv. Dorado, sin embargo, Santana et al. (2011) reportan para durazno 'Douradão' una tasa de respiración de $95 \mathrm{mg} \mathrm{CO}_{2} \mathrm{~kg}^{-1} \mathrm{~h}^{-1}$ al inicio de la maduración, y valores entre $120-130 \mathrm{mg} \mathrm{CO}_{2} \mathrm{~kg}^{-1} \mathrm{~h}^{-1}$ a medida que avanzó el proceso de maduración, resultados que son superiores a los que se obtuvieron en este estudio debido posiblemente a diferencias en la temperatura de almacenamiento.

La pérdida de peso mostró un aumento lineal (Fig. 1B), comportamiento similar al encontrado por Gao et al. (2016) para los cv. Shahong y Oinmi. Al final del periodo de almacenamiento (día 11) se presentó una pérdida de 17,19 $\pm 1,1 \%$ (Fig. 1B), valor que supera a los reportados por Victoria-Escamilla et al. (2013) para los cv. Paquimé $(4,65 \%)$ y Oro Tlaxcala $(4,65 \%)$ y que pone en evidencia la alta perecibilidad del fruto de durazno 'Dorado', ya que de acuerdo con Burg (2004), dependiendo de la especie, el producto pierde su frescura cuando transpira más del 3 al 10\% de su peso fresco. De acuerdo con los resultados, en condiciones del trópico alto colombiano, el fruto del cv. Dorado sin ningún tratamiento poscosecha debería comercializarse y consumirse en la primera semana de cosechado. García (2006) afirma que la pérdida de peso en durazno se debe principalmente a la deshidratación del fruto, generada por el proceso de transpiración, lo cual a su vez, incide en el deterioro del fruto, debido a que acelera la maduración y senescencia (Kader, 2002). Además, Tucker (2014) menciona que durante la maduración, el fruto presenta degradación de la fracción de pectina que debilita las paredes celulares, generándole mayores posibilidades de perder agua, y por ende peso. De acuerdo con Kays y Paull (2004) el consumo de sustratos por respiración aunque en menor medida, también es una causa de la pérdida de peso del fruto.

La firmeza presentó un comportamiento lineal decreciente, al pasar de $12,8 \pm 1,2 \mathrm{~N}$ en el día 1 de almacenamiento a $6,5 \pm 0,41 \mathrm{~N}$ en el día 11 (Fig. 2A), valores considerablemente inferiores a los reportados por Cascales et al. (2005) para la variedad Caterin con un cambio en la firmeza de 65 a $22 \mathrm{~N}$. En frutos de Prunus salicina Lindl (Rozo-Romero et al., 2015) y de duraznos 'Jinli' (Gong et al., 2015) también se reportó disminución de la firmeza durante la maduración.

En general, el ablandamiento del fruto se da como un proceso de modificaciones en la estructura de la pared celular donde se presenta despolimerización de glucanos y solubilización de pectina (D'Ambrosio et al., 2013), y donde intervienen enzimas como poligalacturonasa (PG), pectinmetilesterasa (PME), endo-1,4- $\beta$-glucanasa, $\alpha$-arabinosidasa y $\beta$-galactosidasa (Rodríguez-Félix et al., 2011), entre otras. La degradación en la pared celular genera mayor susceptibilidad del fruto a la pérdida de agua por transpiración, por lo cual también se ve afectado el peso del fruto y su calidad. En durazno el ablandamiento se presenta de forma prematura, debido a que es un cambio drástico que se produce en poco tiempo y genera las mayores pérdidas poscosecha, pues el debilitamiento de la estructura del fruto puede generar mayor susceptibilidad al daño mecánico y al ataque de patógenos (Di Santo et al., 2009), y es una de las principales
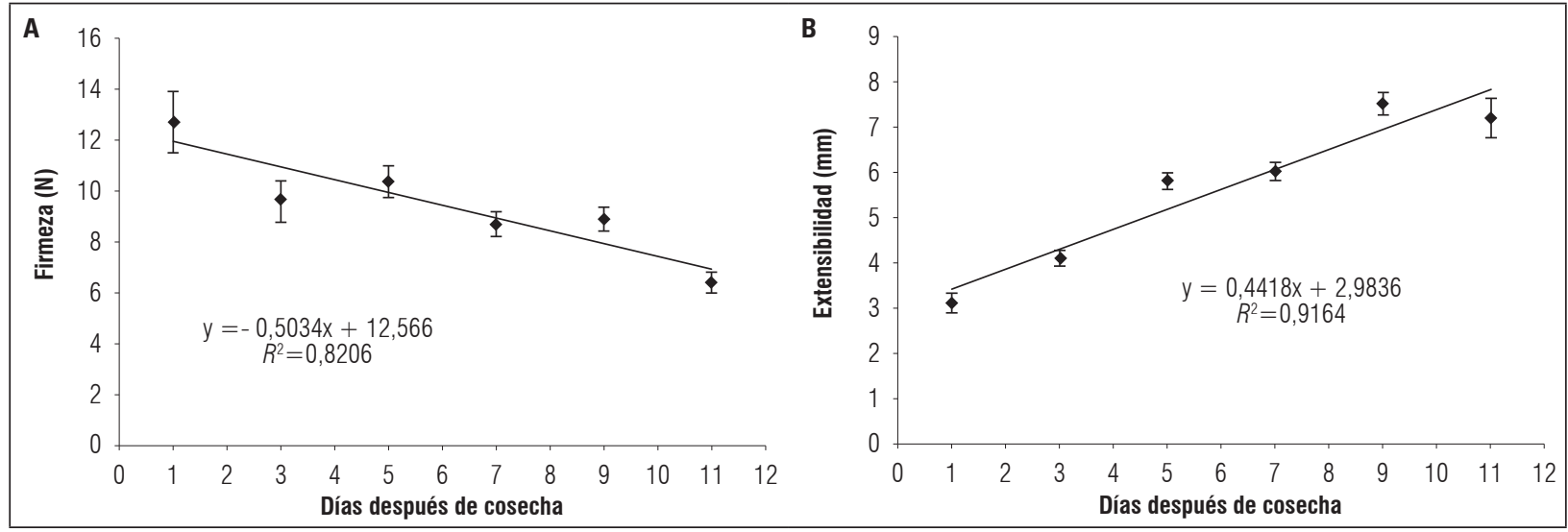

Figura 2. Comportamiento de la firmeza (A) y de la extensibilidad (B) durante la poscosecha del fruto de durazno [Prunus persica (L.) Batsch] cv. Dorado. Los valores corresponden al promedio $(n=4)$. Las barras verticales corresponden al error estándar. 
limitantes en la comercialización del fruto de durazno cv. Dorado.

La extensibilidad aumentó durante el periodo poscosecha al pasar de 3,12 $\pm 0,21$ a 7,21 $\pm 0,44 \mathrm{~mm}$ (Fig. 2B). El aumento de la extensibilidad se debe posiblemente a que durante la fase de maduración la pérdida de agua hace que la epidermis gane flexibilidad (Laguado et al., 1999), García (2016) también observó un aumento de la extensibilidad de la epidermis durante la poscosecha de los frutos de banano bocadillo (Musa acuminata AA, Simmonds), y con valores similares a los encontrados en durazno cv. Dorado.

El índice de color de la epidermis y de la pulpa aumentó durante la maduración con un comportamiento lineal y cuadrático, respectivamente (Fig. 3). Dicho aumento indica cambios de color de la epidermis de tonalidades verdes a rojas y amarillas, mientras que en la pulpa, el cambio de color fue debido al aumento de la intensidad del color amarillo (típico de la variedad). Resultados similares han sido reportados por Cunha et al. (2007) en la variedad Aurora-1. Al respecto, el cambio de color es debido a la degradación enzimática de clorofilas, síntesis de carotenoides y antocianinas y por modificaciones en el pH celular (Slaughter et al., 2013).

En cuanto al color de la pulpa, Slaughter et al. (2013) encontraron un cambio en el color de la pulpa de duraznos cv. Clingstone que pasaron de color amarillo-verdoso a naranja intenso en la maduración, este cambio también está dado por la degradación de la clorofila y la síntesis de carotenoides. Ferrer et al. (2005) también observaron una evolución en el color de la pulpa de duraznos cv. Calanda durante la maduración, dada por la presencia de pigmentos de color amarillo y un menor contenido de clorofilas.

El contenido de SST se ajustó a un modelo cuadrático; se observó que los SST presentaron un aumento

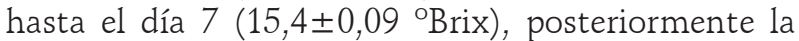
concentración de SST descendió y alcanzó un valor de 14,9 $\pm 0,04$ (Fig. 4A). Los valores de SST indican que el durazno es un fruto dulce, en relación a esto Crisosto et al. (2013) afirman que estos frutos son los que cuentan con mayor aceptación por parte de los consumidores en relación a frutos con menor valor de ${ }^{\circ}$ Brix. En la maduración, el aumento de los sólidos solubles totales se derivan principalmente de la hidrólisis del almidón y polisacáridos de la pared celular en azúcares solubles (sacarosa, glucosa, fructosa y en menores cantidades sorbitol), incrementándose el sabor dulce en los frutos y la pérdida de acidez del fruto (Hiwasa y Ezura, 2014; Kays y Paull, 2004).

El comportamiento de los SST durante la maduración es similar al reportado por Rodríguez-Félix et al. (2011) para cv. Flordaprince. Al final del periodo de almacenamiento se presentó una disminución de los SST, lo cual, de acuerdo a Victoria-Escamilla et al. (2013), podría deberse a la degradación de azúcares y otros compuestos orgánicos en la respiración, conlleva a una pérdida de las propiedades organolépticas y en sí a la pérdida de calidad del fruto.

La ATT disminuyó durante el almacenamiento siguiendo un modelo logarítmico. Al final del periodo poscosecha la acidez fue a $0,76 \%$ (Fig. 4B). Un comportamiento parecido fue reportado para durazno cv.
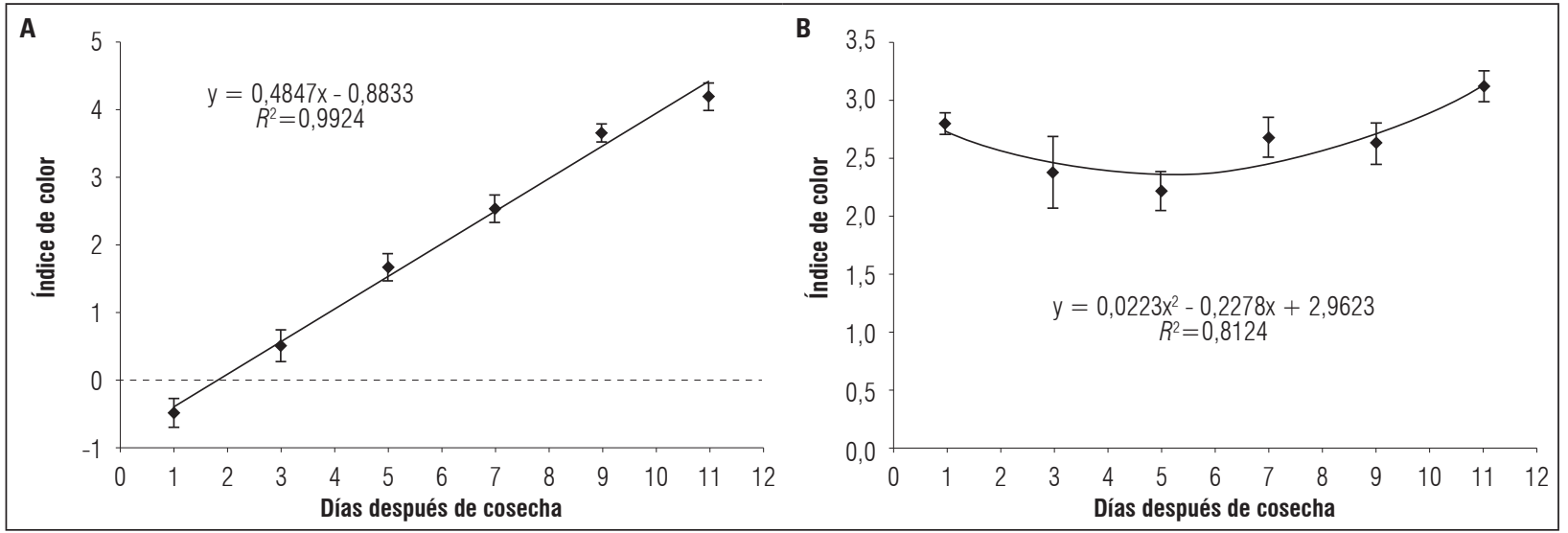

Figura 3. Comportamiento del color de la epidermis (A) y de la pulpa (B) durante la poscosecha del fruto de durazno [Prunus persica (L.) Bastch] cv. Dorado. Los valores corresponden al promedio $(n=4)$. Las barras verticales corresponden al error estándar. 

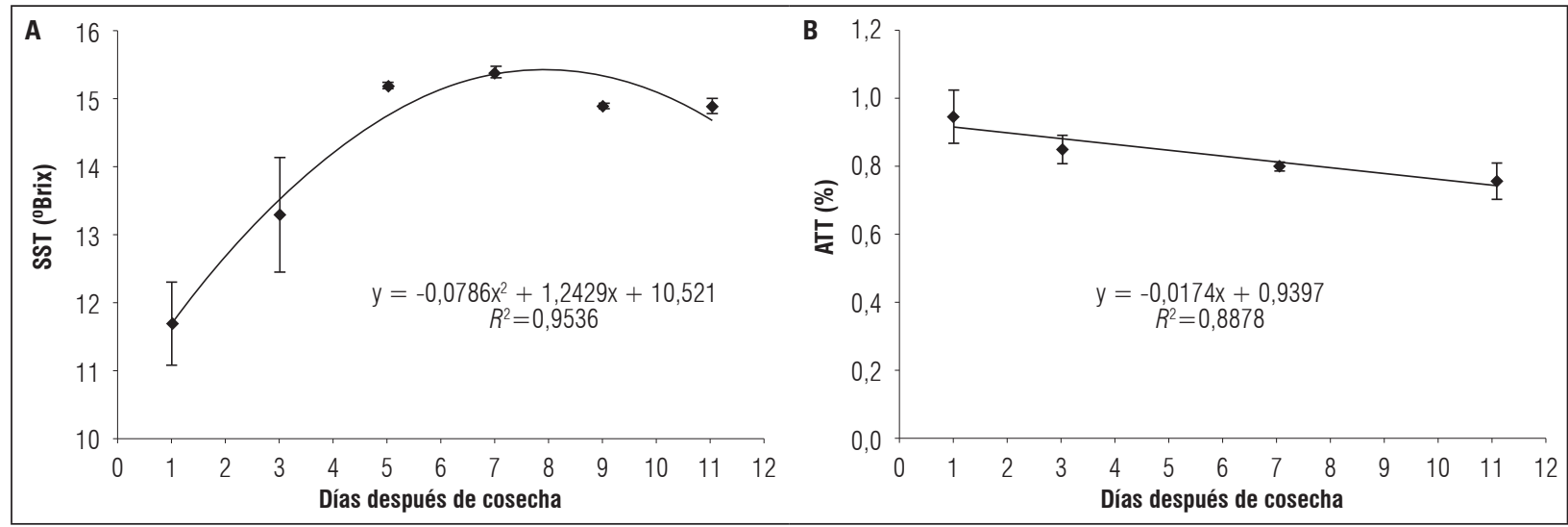

Figura 4. Comportamiento de los sólidos solubles totales (SST) (A) y la acidez total titulable (ATT) (B) durante la poscosecha del fruto de durazno [Prunus persica (L.) Batsch] cv. Dorado. Los valores corresponden al promedio $(n=4)$. Las barras verticales corresponden al error estándar.

Flordaprince (Rodríguez-Félix et al., 2011). Durante la maduración del fruto de durazno, los ácidos orgánicos predominantes son ácido málico y ácido cítrico; estos disminuyen a medida que el fruto madura (Rodríguez-Félix et al., 2011; Day et al., 1997; Wu et al., 2003), debido a que los ácidos orgánicos son usados como sustratos respiratorios por el fruto, y además, pueden ser convertidos en azúcares mediante gluconeogénesis.

La relación de madurez (RM) aumentó durante el tiempo de almacenamiento al pasar de 12,41 a 19,87 (Fig. 5) debido al incremento en los SST y a la disminución de la ATT, esta misma tendencia es reportada por Ferrer et al. (2005) en duraznos cv. Calanda. Por otro lado, el índice de madurez encontrado en este estudio, es menor que el reportado por García (2006) de 41,36. De acuerdo con Defilippi et al. (2011) la relación de madurez está relacionada con la calidad y la aceptabilidad de un fruto por parte de los consumidores. En el fruto de durazno, el sabor junto con el aroma son de las características más importantes que se relacionan con la calidad (Visai y Vanoli, 1997); los carbohidratos cumplen un papel importante en este punto, ya que están directamente relacionados con el sabor del fruto de durazno, el cual está determinado por los azúcares y ácidos orgánicos que el fruto acumule y con la relación del contenido de los azúcares acumulados (Desnoues et al., 2014; Kanayama et al., 2005). Es así como durante la maduración el fruto adquiere un sabor dulce, generado por el aumento del contenido de los SST y la disminución de la ATT (Altube et al., 2001; Rodríguez-Félix et al., 2011).

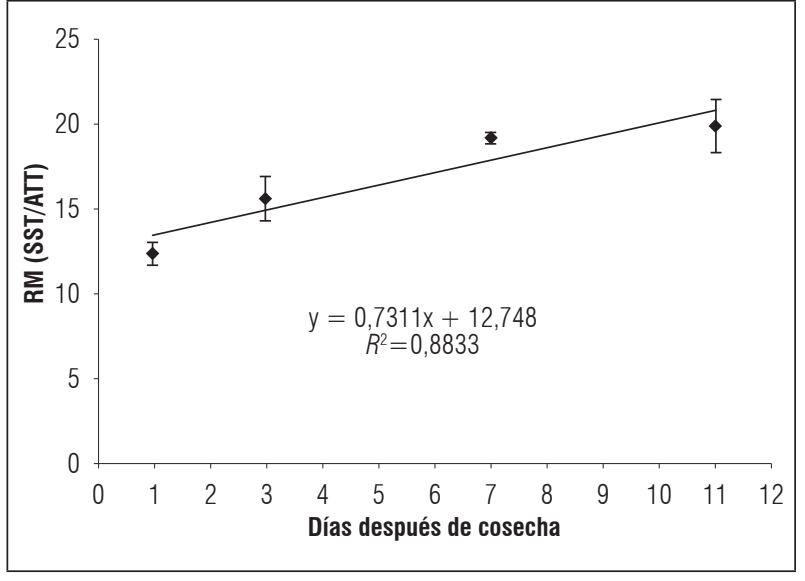

Figura 5. Relación de madurez (RM) durante la poscosecha del fruto de durazno [Prunus persica (L.) Batsch] cv. Dorado. Los valores corresponden al promedio $(n=4)$. Las barras verticales corresponden al error estándar.

\section{CONCLUSIONES}

El fruto de durazno cv. Dorado, producido en condiciones del trópico, es un fruto climatérico que mostró un incremento en la tasa respiratoria después de que alcanzó la madurez fisiológica. En la maduración del fruto se presentaron pérdida del color verde de la epidermis, pérdida de peso, disminución de la firmeza y de la acidez total titulable e incremento en la concentración de sólidos solubles totales y la relación de madurez. 


\section{REFERENCIAS BIBLIOGRÁFICAS}

Africano, K., P.J. Almanza y H.E. Balaguera-López. 2015. Fisiología y bioquímica de la maduración del fruto de durazno [Prunus persica (L.) Batsch]. Una Revisión. Rev. Colomb. Cienc. Hortic. 9(1), 161-172. Doi: 10.17584/rcch.2015v9i1.3754

Agronet. 2016. Área, producción y rendimiento de durazno en Colombia. En: http://www.agronet.gov.co; consulta: junio de 2016.

Akbudak, B. y A. Eris. 2004. Physical and chemical changes in peaches and nectarines during the modified atmosphere storage. Food Control 15, 307-313. Doi: 10.1016/S0956-7135(03)00082-3

Altube, H., C. Budde, M. Ontivero y R. Rivata. 2001. Determinación de los índices de cosecha de duraznos cvs. Flordaking y San Pedro 16-33. Agric. Tec. 61(2), 140150. Doi: $10.4067 / s 0365-28072001000200004$

Balaguera-López, H., C. Martínez y A. Arévalo. 2014. Papel del cáliz en el comportamiento poscosecha de frutos de uchuva (Physalis peruviana L.) ecotipo Colombia. Rev. Colomb. Cienc. Hortic. 8(2), 181-191. Doi: 10.17584/ rcch.2014v8i2.3212

Balaguera-López, H., M. Espinal-Ruiz, L. Zacarías y A. Herrera. 2016. Effect of ethylene and 1-methylcyclopropene on the postharvest behavior of cape gooseberry fruits (Physalis peruviana L.). Food Sci. Technol. Int. Doi: $10.1177 / 1082013216658581$

Brovelli, E., J. Brecht, W. Sherman y C. Sims 1999. Nonmelting-flesh trait in peaches is not related to low ethylene production rates. HortScience 34(2), 313-315.

Burg, S.P. 2004. Postharvest physiology and hypobaric storage of fresh products. CABI Publishing, Wallingford, UK. Doi: 10.1079/9780851998015.0000

Campos, T. 2013. Especies y variedades de hoja caduca en Colombia. pp. 47-67. En: Miranda, D., G. Fischer y C. Carranza (eds.). Los frutales caducifolios en Colombia. Situación actual, sistemas de cultivo y plan de desarrollo. Sociedad Colombiana de Ciencias Hortícolas, Bogotá.

Cascales, A., E. Costell y F. Romojaro. 2005. Effects of the degree of maturity on the chemical composition, physical characteristics and sensory attributes of peach (Prunus persica) cv. Caterin. Food Sci. Technol. Int. 11(5), 345-352. Doi: 10.1177/1082013205057943

Castro, A. y G. Puentes. 2012. Ciruelo y duraznero (Prunus salicina Lindl) - (Prunus pérsica (L.) Batsch). pp. 370392. En: Fischer, G. (ed.). Manual para el cultivo de frutales en el trópico. Produmedios, Bogotá.

Crisosto, C., E. Mitcham y A. Kader 2013. Nectarine \& peach: Recommendations for maintaining postharvest quality. En: http://postharvest.ucdavis.edu; consulta: noviembre de 2016 .
Cunha, L., M. Berlingieri, B. Mattiuz, R. Nogueira y J. Durigan 2007. Caracterização da curva de maturação de pêssegos 'Aurora-1', na região de Jaboticabal-Sp. Rev. Bras. Frutic. 29(3), 661-665. Doi: 10.1590/ S0100-29452007000300045

D’Ambrosio, C., S. Arena M. Rocco F. Verrillo, G. Novi, V. Viscosi, M. Marra y A. Scaloni. 2013. Proteomic analysis of apricot fruit during ripening. J. Proteomics 78, 39-57. Doi: 10.1016/j.jprot.2012.11.008

Day, K., G. Crisosto, C. Crisosto y M. Watkins. 1997. Survey of white flesh nectarines and peaches. Fruit Varieties J. 52, 184-189.

Defilippi, B., C. Becerra, I. Salgado y P. Robledo 2011. Comportamiento en poscosecha de variedades de carozo producidas en Chile y destinadas a mercados de exportación. pp. 105-116. En: Lemus G. e I. Salgado. Centro de Frutales de Carozo: Resumen Técnico. Boletín INIA 227. Chile.

Desnoues, E., Y. Gibon, V. Baldazzi, V. Signoret, M. Génard y B. Quilot-Turion. 2014. Profiling sugar metabolism during fruit development in a peach progeny with different fructose-to-glucose ratios. BMC Plant Biol. 14:336. Doi: 10.1186/s12870-014-0336-x

Di Santo, C., E. Pagano y G. Sozzi. 2009. Differential expression of $\alpha$-L-arabinofuranosidase and $\alpha$-L-arabinofuranosidase/ $\beta$ - $D$-xylosidase genes during peach growth and ripening. Plant Physiol. Bioch. 47, 562569. Doi: 10.1016/j.plaphy.2009.02.007

Ferrer, A., S. Remón, A. Negueruela y R. Oria. 2005. Changes during the ripening of the very late season Spanish peach cultivar Calanda: Feasibility of using CIELAB coordinates as maturity indices. Sci. Hortic. 105(4), 435-446. Doi: 10.1016/j.scienta.2005.02.002

Fischer, G., F. Casierra-Posada y C. Villamizar. 2010. Producción forzada de duraznero (Prunus persica (L.) Batsch) en el altiplano tropical de Boyacá (Colombia). Rev. Colomb. Cienc. Hortíc. 4(1), 19-32. Doi: 10.17584/ rcch.2010v4i1.1223

Gao, H., Z. Zhang, H. Chai, N. Cheng, Y. Yang, D. Wang, T. Yang y W. Cao. 2016. Melatonin treatment delays postharvest senescence and regulates reactive oxygen species metabolism in peach fruit. Postharvest Biol. Technol. 118, 103-110. Doi: 10.1016/j. postharvbio.2016.03.006

García A. 2006. Caracterización física y química de duraznos (Prunus persica (L.) Batsch) y efectividad de la refrigeración comercial en frutos acondicionados. Bioagro 18(2), 115-121.

García, J.C. 2016. Efecto del 1-Metilciclopropeno (1-MCP) en el comportamiento poscosecha de banano bocadillo (Musa acuminata AA, Simmonds). Tesis de maestría. Facultad de Ciencias Agrarias, Universidad Nacional de Colombia, Bogotá.

Gong, D., S. Cao, J. Shao, C. Song, F. Wo, W. Chen y Z. Yang. 2015. Effect of blue light on ethylene biosynthesis, signalling and fruit ripening in postharvest 
peaches. Sci. Hortic. 197, 657-664. Doi: 10.1016/j. scienta.2015.10.034

Herrera, C., M. Laitón, A. Paredes y G. Sánchez. 2006. Manejo poscosecha en durazno y ciruelo. En: Corporación Colombiana de Investigaciones Agropecuaria (Corpoica). Produmedios, Bogotá.

Hiwasa, K y H. Ezura. 2014. Physiology and metabolism. pp 1-98. En: Nath, P., M. Bouzayen, J. Pech y A. Mattoo (eds). Fruit ripening: physiology, signalling and genomics. CABI, London.

Infante, R., L. Contador, P. Rubio, D. Aros y A. Peña-Neira. 2011. Postharvest sensory and phenolic characterization of 'Elegant Lady' and 'Carson' peaches. Chil. J. Agric. Res. 71(3), 445-451. Doi: 10.4067/ S0718-58392011000300016

Kader, A.A. 2002. Standardization and inspection of fresh fruits and vegetables. pp. 287-314. En: Kader, A.A. (ed.). Postharvest technology of horticultural crops. $3^{\text {rd }}$ ed. Ed. University of California, Division of Agriculture and Natural Resources, Oakland, CA.

Kanayama, Y., M. Kogawa, M. Yamaguchi y K. Kanahama. 2005. Fructose content and the activity of fructose related enzymes in the fruit of eating - quality peach cultivars and native - type peach cultivars. Jpn. Soc. Hortic. Sci. 74, 431-436. Doi: 10.2503/jjshs.74.431

Kays, S.J. y R.E. Paull. 2004. Postharvest biology. Exon Press, Athens, GA.

Laguado, N., E. Pérez, C. Alvarado y M. Marín. 1999. Características fisicoquímicas y fisiológicas de frutos de guayaba de los tipos Criolla Roja y San Miguel procedentes de dos plantaciones comerciales. Rev. Fac. Agron. (LUZ) 16, 382-397.

Lambaré, A. y L. Pochettino. 2012. Diversidad local y prácticas agrícolas asociadas al cultivo tradicional de duraznos, Prunus persica (Rosaceae), en el noroeste de Argentina. Darwiniana 50(2), 174-186.

Liu, H., C. Jiankang y W. Jiang. 2015. Changes in phenolics and antioxidant property of peach fruit during ripening and responses to 1-methylcyclopropene. Postharvest Biol. Technol. 108, 111-118. Doi:10.1016/j. postharvbio.2015.06.012

Miranda, D. y C. Carranza. 2013. Caracterización, clasificación y tipificación de los sistemas productivos de caducifolios con énfasis en duraznero, manzano, ciruelo y peral. pp. 87-105. En: Miranda, D., G. Fischer y C. Carranza (eds.). Los frutales caducifolios en Colombia. Situación actual, sistemas de cultivo y plan de desarrollo. Sociedad Colombiana de Ciencias Hortícolas, Bogotá.

Pinzón, E., A.C. Morillo y G. Fischer. 2014. Aspectos fisiológicos del duraznero (Prunus persica [L.] Batsch) en el trópico alto. Una revisión. Rev. UDCA Actual. Divulg. Cient. 17(2), 401-411.

Puentes, G., L. Rodríguez y L. Bermúdez. 2008. Análisis de grupo de las empresas productoras de frutales caducifolios del departamento de Boyacá. Agron. Colomb. 26(1), 146-154.
Rodríguez-Félix, A., J. Fortiz-Hernández y M. Villegas-Ochoa. 2011. Cambios en enzimas pectolíticas durante la maduración del durazno 'Flordaprince'. Interciencia 36(1), 65-70.

Rozo-Romero, X., J. Álvarez-Herrera y H.E. Balaguera-López. 2015. Ethylene and changes during ripening in 'Horvin' plum (Prunus salicina Lindl.) fruits. Agron. Colomb. 33(2), 228-237. Doi: 10.15446/agron.colomb. v33n2.49856

Ruiz-Altisent, M., L. Lleó y F. Riquelme. 2006. Instrumental quality assessment of peaches: fusion of optical and mechanical parameters. J. Food Eng. 74, 490-499. Doi:10.1016/j.jfoodeng.2005.01.048

Santana, L., B. Benedetti y J. Sigrist. 2011. Effects of modified atmosphere packaging on ripening of 'Douradão' peach related to pectolytic enzymes activities and chilling injury symptoms. Rev. Bras. Frutic. 33(4), 1084 1094. Doi: 10.1590/S0100-29452011000400006

Slaughter, D.C., C.H. Crisosto y G. Tiwari. 2013. Nondestructive determination of flesh color in clingstone peaches. J. Food Eng. 116, 920-925. Doi:10.1016/j. jfoodeng.2013.01.007

Toncovich, M., C. Quinteros, C. Uriona, P. Ortín, M. Tejerina, A. Tálamo y A. González 2008. Análisis de la calidad de fruto a cosecha de nuevas variedades de duraznero (Prunus persica) en el Valle de Lerma (Salta). Hort. Arg. 27(64), 101.

Tonetto De Freitas, S., C. Sautter, A. Silveira y A. Brackmann. 2007. Evaluación del efecto de la aplicación de 1-mcp y la conservación en atmósfera controlada sobre la calidad poscosecha de duraznos en dos estados de madurez. Agrociencia 11, 67-72.

Tucker, M. 2014. Cell-wall metabolism and softening during ripening. Pp. 48-62. En: Nath, P., M. Bouzayen, J. Pech y A. Mattoo (eds.). Fruit ripening: physiology, signalling and genomics. CABI, London.

Victoria-Escamilla, M., S. Chaves, Y. Mendoza, J. Molina y D. Sangerman- Jarquín. 2013. Diseño, fabricación y evaluación del prototipo de simulación de esfuerzos dinámicos en durazno (Prunus persica). Rev. Mex. Cien. Agric. 4(6), 933-945.

Visai, C. y M. Vanoli. 1997. Volatile compound production during growth and ripening of peaches and nectarines. Sci. Hortic. 70(1), 15-24. Doi: 10.1016/ S0304-4238(97)00032-0

Weber, M., D. Güemes, M. Pirovani, A. Piagentini, A. Zanuttini y N. Gariglio 2003. Características del fruto del duraznero 'Flordaking' cultivado en la zona centro-este de la provincia de Santa Fe (Argentina). Cienc. Agrar. 2(1-2), 29-35.

Wu, B., B. Quilot, J. Kervella, M. Génard y S. Li. 2003. Analysis of genotypic variation of sugar and acid contents in peaches and nectarines through the principle component analysis. Euphytica 132, 375-384. Doi: 10.1023/A:1025089809421 